\title{
Computational and mathematical approaches to societal transitions
}

\author{
Jos Timmermans • Hans de Haan • \\ Flaminio Squazzoni
}

Published online: 20 September 2008

(C) The Author(s) 2008. This article is published with open access at Springerlink.com

\begin{abstract}
After an introduction of the theoretical framework and concepts of transition studies, this article gives an overview of how structural change in social systems has been studied from various disciplinary perspectives. This overview first leads to the conclusion that computational and mathematical approaches and their practical form, modeling, up till now, have been almost absent in the research and theorizing of structural change or transitions in social systems. Second, this review of the social science literature suggests numerous theoretical constructs relevant for transition modeling. Relevant concepts include the conceptualization of the micro-tomacro link, the importance of explaining both stability and change, quantitative and qualitative definitions of structural change, the use of dichotomies, synchronic and diachronic reasoning in explaining structural change, definitions of basic patterns of social change, the conceptualization of resistance to change and intentional and normative aspects of social change. This article employs these theoretical concepts to describe and discuss the models presented in this special issue in order to develop an understanding of what exactly entails a computational or mathematical approach to societal transitions.
\end{abstract}

\footnotetext{
J. Timmermans $(\bowtie) \cdot$ J. de Haan

Faculty of Social Sciences, Dutch Research Institute for Transitions, Erasmus University Rotterdam, P.O. Box 1738, 3000 DR, Rotterdam, The Netherlands

e-mail: timmermans@fsw.eur.nl

url: http://www.eur.nl/fsw/staff/homepages/timmermans/

J. de Haan

e-mail: j.dehaan@fsw.eur.nl

url: http://www.eur.nl/fsw/staff/homepages/dehaan/

F. Squazzoni

Department of Social Sciences, University of Brescia, Via S. Faustino, 74/B, 25122 Brescia, Italy

e-mail: squazzon@eco.unibs.it

url: http://www.unibs.it/on-line/dss/Home/Personale/PersonaleDocente/articolo1758.html
} 
Keywords Societal transitions · Micro-macro link · Agent-based models · Complexity

\section{Introduction}

Although much of the conceptual language is taken from complexity theories, which have strong connections with exact sciences, computational and mathematical approaches are hard to find in the field of transition studies. It is the strong conviction of the authors, and of all the authors in this special issue, that computational and mathematical methods can further a science in a way other methods can not. The practical form of applying such methods, e.g. modeling, forces the researchers to be strict in their reasoning. Not that a mathematical model is always right, but at least it can be exactly wrong.

Mathematical models are suitable to grasp the working mechanisms behind patterns of natural and social life, as well as to test and extend theoretical findings. Computational methods are useful to formalize complex models that are difficult, if not impossible, to treat with standard analytic methods, because of non linearity, heterogeneous agents, and local interactions that are involved in the system under observation (Gilbert 2008). As a matter of fact, the relevance of formalization significantly increases when phenomena to be studied require collaboration among scientists belonging to different scientific disciplines. This is a further reason why computational and mathematical approaches are really necessary for the progress of transition studies.

But what exactly is a transition model? Is it any model modeling a societal transition? At first glance there is nothing to be said about what transition modeling entails. It appears that all methods are allowed and all approaches have their merits and shortcomings. This issue alone already covers five methodologically different approaches to societal transitions; agent based modeling, partial differential equations, mathematical sociology, system dynamics and non-linear systems. Other promising approaches like cellular automata, evolutionary modeling, nk-modeling or binary networks are even not included.

The research question formulated above is approached by first presenting a review of the social science literature on structural change. In this extensive literature theoretical constructs are identified that are pertinent to structural change. These theoretical constructs are then used to describe, compare and discuss the conceptualization of change in the models presented in this issue and so develop a better understanding of what actually entails a transition model.

\section{Societal transitions}

Apart from disciplinary approaches to transitions, in the last decade societal transitions have become a research topic in themselves. It was in fact two strands of research stumbling upon each other that made this field take shape. The first strand was that of socio-technical transitions, where transitions are studied that revolve around 
some technology like the transition from horse-car to automobile (Geels 2005). The other strand was that of integrated assessment which aims to combine knowledge from various scientific disciplines to assess societal issues (Rotmans 1998). The convergence of these strands in a field that could be called 'transition studies' was led by the realization that many of the current societal systems suffer from persistent problems and that transitions are necessary to let them continue developing in a more sustainable direction.

Transition studies conceptualize societal transitions as processes of fundamental change in the structures, cultures and practices of societal systems. These changes are considered to be long term, that is, several generations, and large scale (Rotmans 2005). What a societal system actually entails, is left rather open, in general are meant sector systems like energy supply systems or mobility systems (e.g. Berkhout et al. 2004; Verbong and Geels 2006), but also regional systems like cities (Byrne 2001) or ports (Driel and Schot 2005), transitions in health care systems and water management systems (e.g. van der Brugge and Rotmans 2006) have also been studied in this manner.

To get a conceptual grip on the multi-faceted processes of transitions, refuge is sought in complexity theories leading to a societal system being conceptualized as a complex adaptive system moving from one dynamical equilibrium to another (Rotmans 2005). Combined with the terminology from socio-technical transitions one obtains the conceptualization of a societal transition as a shift of 'regime' in a societal system (Rip and Kemp 1998). A regime in this view epitomizes the dominant way the system functions, in terms of structures, cultures and practices. The environment of a societal system is called the 'landscape' and changes in the landscape provide a climate for change, while the concept of a nucleus of novel or deviant functioning, which is referred to as a niche, furnishes alternatives to the dominant functioning or regime (Fig. 1).

This multi-level picture of regime, niche and landscape has been introduced by Rip and Kemp (1998) and was subsequently elaborated much by Geels (Geels and Schot 2007). Further theoretical developments have been introduced by Rotmans,

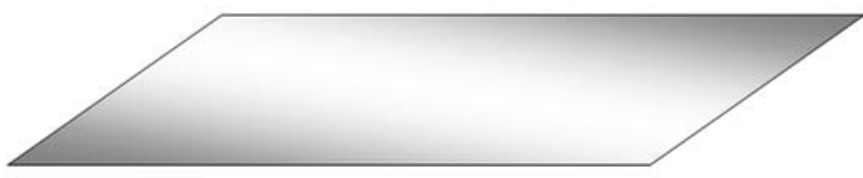

Macro

Where the landscape resides, the environment of the societal system. Exogenous influences, long term developments.

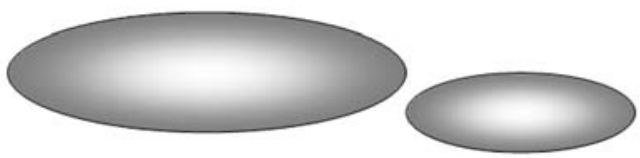

Meso

Primary level of analysis and basis for system demarcation. Level of the regime and possible niche-regimes.
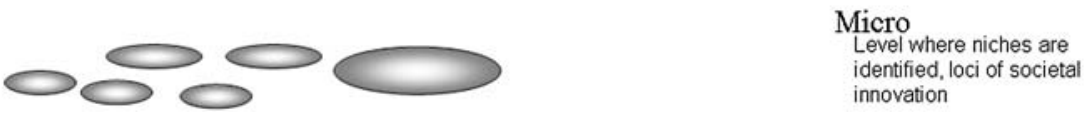

Fig. 1 Multi-level model of societal transitions 


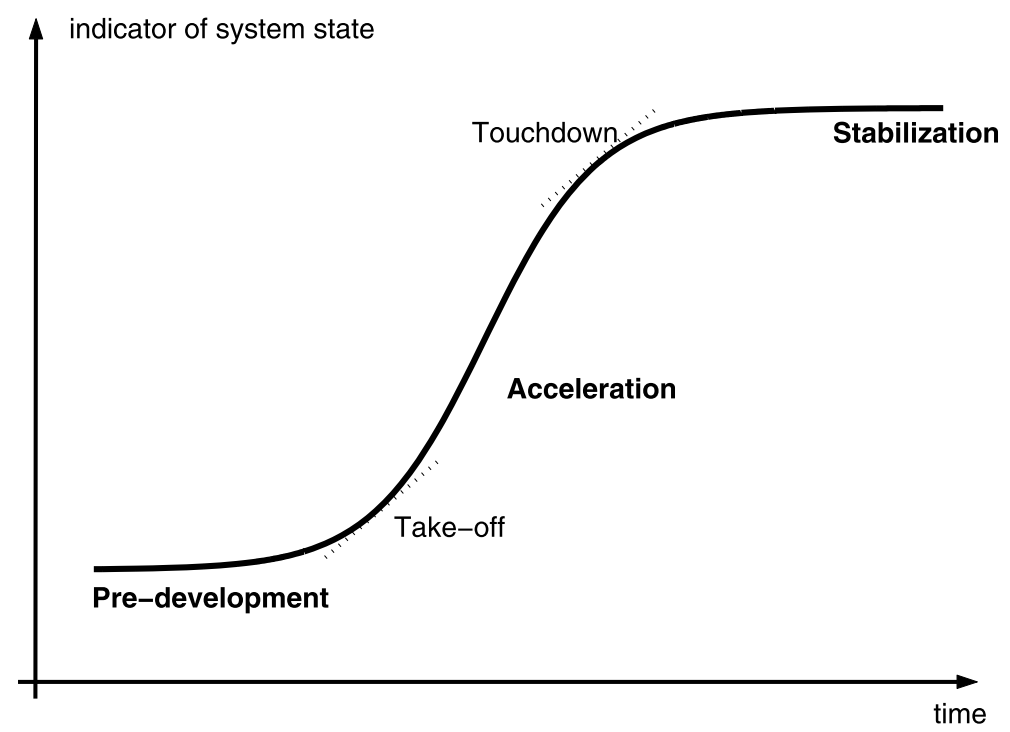

Fig. 2 Multi-phase model of societal transitions

who uses a similar multi-level model identifying a micro, meso and macro level and who distinguishes multiple phases in transitions, visualized by an s-shaped curve in analogy to innovation diffusion and the demographic transition (Davis 1945; Rotmans et al. 2000). The multi-level model is often accompanied by the concept of 'modulation', the idea that developments on regime-niche-landscape levels have to go in the same direction to make a transition possible. That the societal system is somehow resisting a transition is often discussed in terms of the regime being in a 'lock-in' situation, which is the result of path-dependent developments in building up the current status quo amplified by vested interests.

To distinguish transitional change from general societal change the multi-phase picture is often employed. In analogy to the phases of innovation diffusion and the demographic transition an S-shaped curve runs from the 'beginning' to the 'end' of a transition subsequently going through a predevelopment phase via a take-off into an acceleration phase to touch down in a stabilization phase (Fig. 2). This alternation of relatively slow and fast dynamics in the societal change is considered typical for a transition.

\section{Structural change in social systems}

The use of the concept of transition in the study of societal transitions is distinct though related to its use in the social sciences. At the same time the link with complexity theory gives the concept of societal transitions a natural science flavor. Similar interdisciplinary connections exist between transition studies and the policy sciences, which are related both via complexity theory and the governance literature. This section further defines the field of societal transitions through its positioning in 
the context of 'approaches to transitions' from other fields of social science. We do so by working from small to big social scales, starting with the organizational sciences and ending with sociology and by confronting approaches and concepts of structural change in the social science literature with the concepts from the field of societal transitions described in Sect. 2. In addition a short detour to the natural sciences is offered.

\subsection{Natural sciences}

To look at structural change or transitions from a natural science perspective two angles are available. Firstly the actual word 'transition' is used quite often, signifying different phenomena but in general with the common denominator of a rather abrupt change of state, like a spectral transition where an atom instantly goes into a state of lower energy, emitting a photon. Often the change of state is not only abrupt but also qualitative, like in phase transitions as for example the transition from water to ice. Secondly, there is the host of phenomena that are or are not named transitions in natural science akin to societal transitions in that they share more than just being about, abrupt, qualitative change.

Amongst the phenomena that have similar characteristics as the societal transition studied in this issue some deserve mentioning here since their similarity introduced some natural science terminology into the language of transition research. The evolutionary notion of punctuated equilibrium (Eldredge and Gould 1972; Gould and Eldredge 1977) for instance, where long periods of gradual evolution are separated by sudden bursts of change after which gradual evolution continues. This notion has already been taken up by the organizational sciences and appears useful for transition research. Ecologists developed the panarchy and resilience approach to the complexity of ecological systems which appears as applicable to societal systems as well (Gunderson and Holing 2002). On a more metaphorical level the ideas that societal systems are dissipative structures operating at the edge of chaos and that transitions can be considered attractor changes, have been put forth (Byrne 1998). It would be worthwhile to explore how far these metaphors hold and if they can be of help in furthering the field of transition studies as a science.

Other lines of thought that have not been explored in this issue, but that might be interesting, are for instance some ideas that have been popular but appear to be on the decline in recent times like the thermo economics of Georgescu-Roegen (1971) that has become a theoretical anchor in fields like ecological economics. Especially when combined with Prigogine's (Nicolis and Prigogine 1989) ideas on dissipative structures and far-from-equilibrium thermodynamics, this appears a fruitful angle for novel computational and mathematical approaches. These works, however exact, hover on a high level of abstraction which poses difficulties in applying them straightforwardly. Something similar holds for the work of Wolfram (2002) concerning cellular automata.

\subsection{Organizational sciences}

In the organizational sciences, more specific the field of organizational adaptation, Miller and Friesen introduce the concept of organizational transition. Using environmental, structural and strategy-making characteristics they developed nine archetypes 
of organizational transitions (Miller and Friesen 1984). Miller and Friesen maintain that organizational transitions result from an imbalance or stress resulting from a key event or discussion and unfolds as 'a package of changes that occur between the onset of the imbalance or stress and the time when some equilibrium or tranquil interval is reached' (Miller and Friesen 1984). In transition studies similar phase delimiters are labeled take off, acceleration and stabilization (van der Brugge and Rotmans 2006). In their operational definition Miller and Friesen delimit an organizational transition as the period in between two tranquil intervals (Miller and Friesen 1984).

In reaction to the analytic character and lack of managerial impact of organizational adaptation, change management emerged (Worren et al. 1999). In change management the concept of transition addresses both organizational change and individual change. In individual change transition refers to a confusing period of change in between periods of overcoming inertia and bypassing defense mechanisms, "unfreezing", and internalizing a new mindset, "refreezing" as described in the phase model of Lewin (Lewin 1951). Organizational transitions address the way organizations adapt to a changed environment. In change management organizational transitions are connected to individual transitions in order to manage the 'people site' of organizational change.

The phase model of Lewin is also applied in the leadership theories of the neocharismatic school (Fiol et al. 1999) and is similar in both structure and meaning to the phase model of societal transitions. However, the connection to personal change as made in change management and neo-charismatic leadership theory is not made in transition studies. In transition studies the mindset of an individual is replaced by the concept of culture of specific social sub-systems like regimes or niches. Both transition studies and change management emphasize the need for unfreezing, bypassing inertia, overcoming defense mechanisms, breaking down existing power structures and destabilizing the existing regime (Fiol et al. 1999; Lewin 1951; van der Brugge and Rotmans 2006). Both strands of social research define transitions primarily on qualitative change and relatively fast change in itself is not enough for a specific period to qualify as a transition.

\subsection{Policy sciences}

In the policy sciences the alternation of long periods of stability and incremental change with short periods of violent change is a central issue (Baumgartner and Jones 1993; Jones and Baumgartner 2005; Kingdon 1984; Wilson 2000). The focus is on sudden changes in the otherwise stable processing of issues and the related rapid change in the public policy arrangement around them. Concepts like transition or transformation are not used, but explicit reference to concepts from complex systems theory like punctuated equilibrium are made (Baumgartner and Jones 1993; Jones and Baumgartner 2005). Similar to transition studies, in the policy sciences research ambitions focus on the development of models that are able to both explain long periods of stability and short periods of rapid change. Explanatory models range from political process and decision making models, elitists and neo-Marxist theorizing, explanatory models rooted in postmodern and cultural theory and state centered approaches (Wilson 2000). The policy regime model (Wilson 2000) integrates many 
of the dimensions explored in the approaches above, while introducing the concept of policy regime and regime change to explain the alternation of periods of stability with periods of dramatic change. Policy scientists are inclined to emphasize the role of perturbations, like disasters and inflation in combination with the role of the media and other influences and factors external to the policy system itself. Here, the punctuated equilibrium model of Baumgartner and Jones is a notable exception. In their model Baumgartner and Jones analyze the episodic behavior of policy change as internal to the policy system, a policy system becoming critical through what they describe as a positive feedback between image and venue (Baumgartner and Jones 1993). They also recognize that the period of change itself can be dramatic.

Although the policy sciences do not use the term transition, their theoretical concepts and terminology have much in common with transition studies. It also has its integrative focus on the entire process of policy change, from stability to stability, in common with transition studies. On the other hand, in the policy sciences a focus on resistance to change and the conceptualization of a period of chaos are normally lacking. The definition of dramatic change in the policy sciences is mainly based on the speed of change and not on a qualitative evaluation of change and is normally described using empirical observations like the dissolution of an organization and the installation of another. Some authors in the policy sciences (e.g. Sabatier and JenkinsSmith 1993) emphasize the change itself by endowing policy coalitions with a belief system. Sabatier also uses the notions of dominant and minority coalitions, each with their own belief system, while change is analyzed as the take over of the dominant position by a minority coalition (Sabatier and Jenkins-Smith 1993). Here Sabatier comes very close to the concept of regime and niche used in transition studies.

Like transition theory, the policy regime model (Wilson 2000) integrates factors internal and external to the policy regime, including large scale autonomous developments. However, although the policy regime model and transition studies operate on the same analytical level, the research object of the policy sciences is the processing of societal issues and not a societal system fulfilling a societal function including issue processing related to this function. The policy regime model, or more general 'transition theories' from the policy sciences, are thus insensitive to their own role in creating and maintaining the societal issues they process. It is exactly at this point that transition studies and transition management seek the leverage to address persistent problems out living one policy regime after another.

\subsection{Political science}

In the vast political science literature on transition of political regimes towards democratization, a strong emphasis is put on the role of political elites in molding appropriate institutions for the consolidation of new regimes against traditional institutional systems (Eisenstadt 1973; Linz and Stepan 1996). Classical examples are Black's model of modernization (Black 1966) and Deutsch model of social mobilization and political development (Deutsch 1961). Deutsch focuses on the process of societal mobilization as an "overall process of change, which happens to substantial parts of the population in countries which are moving from traditional to modern way of life". He develops an analytical model and a set of statistical indicators and 
comparative measurements to express the idea that societal change is a "process in which major clusters of old social, economic and psychological commitments are eroded or broken and people become available for new patterns of socialization and behavior" (Deutsch 1961). In conclusion, this literature emphasizes the social conflict behind transitions, their planned and intentional nature, against any emergentist flavor, the relevance of particular social groups in promoting, strengthening and taking the change, like political elites, as well as how past leaders and dominant groups in the society can make a stand against any disruptive change.

\subsection{Economics}

Mainstream economics has not directly addressed or used the notion of transitions However, concepts like development, growth, structural change, (system) innovation and transformation bear close relationship to it (van den Bergh and Kemp 2006). So far only in three areas of economic research the term transition has appeared. In theoretical dynamic models of economic growth, the term transition is used to denote the change from one equilibrium state to another. Furthermore in the study of the transformation of a planned into a market economy in the former communist and Eastern-European countries the term transition has been used (Aassve et al. 2006); The somewhat related research field of development economics uses the term transition in analyzing the conditions under which, poor, rural countries can change into modern market-based economies.

In heterodox economic schools, like evolutionary economics and institutional economics, transitions are integral to the specific paradigms. In evolutionary economics, transitions are interpreted similar to transitions in evolutionary biology, including the concept of increasing complexity and the emergence of new levels of reality. This school uses an evolutionary paradigm and consequently numerous concepts from both evolutionary biology and complex adaptive systems theory, like emergence, transition, co-evolution and punctuated equilibrium (Arthur 1994; Rammel and van den Bergh 2003; van den Bergh and Gowdy 2003; van den Bergh and Stagl 2006; Witt 2003). Potts analyzes economic growth and change essentially as a change in connections between physical entities, material and information flows between individuals or departments within an organization causing new products, new firms and new sectors to rise and old ones to disappear (Potts 2000). Similarly, institutional economics fosters a focus on change, learning and evolution while emphasizing the shaping influence of (economical) institutions on individual action, while transition are related to changes in these institutions (North 2005).

Economic history tends to analyze societal developments on a higher scale level, e.g. entire societies, but starts from a focus on the economic sub-system. Often, however, the impact of the way the economic system is governed is so intimately related to other sectors of a society that the analyses end up with a breadth and depth similar to sociological contributions. Good examples here are Rostow's (1960) description of the transition towards modernity and Marx's stage model of the transition from capitalism to socialism. Besides their initial focus on the economic subsystem, these theories share a linear approach and both envision a stable final state. Although also Schumpeter envisioned a socialist end state, albeit for different reasons, Schumpeter 
maintained that a capitalist economic system can never be stationary. He therefore emphasized social transformation and argued that equilibrium analyses fails to grasp the dynamics of capitalism and the processes by which economic institutions rise and fall (Medearis 2001). Schumpeter's theory is build around the notion of "creative destruction", a process that revolutionizes economic structures from within by destroying old and creating new structures (Schumpeter 1942). In their model of growth through creative destruction, Aghion and Howitt (1992) make clear that this process is forward looking and based on destruction of the existing, while no resistance to change is conceptualized although a negative feedback on research expenditures is envisioned, stemming from the destruction of possible future gains through the earlier arrival of the next innovation (Aghion and Howitt 1992).

Weber is a good example of the close relation between sociology and economic history as he connects economical transformation and the development of specific values rooted in a religious tradition (Weber 1904/1905). The same holds for Karl Polanyi's historical analysis of the "great transformation", that is a transition of Western societies between 18th and 19th century, with the advent of the modern market economy and society through the corresponding institutional change at the edge of political, economic and social systems (Polanyi 1944). This great transformation emphasizes the strength of the diffusion of market institutions across political and social spheres. This same evidence has been presented by the French historian Fernand Braudel, who, in his monumental trilogy on civilization, market economy and capitalism, offers a theoretical perspective on the transition towards modernity based on the idea of long-term continuity and directionality of changes across culture, political, social and economic spheres (Braudel 1979).

Both Polanyi and Braudel, like Schumpeter, emphasize the continuous interplay between groups that are bearers of changes and innovation, on one side, and the breaking strength of dominant groups, which are challenged by the novelties introduced by the firsts, on the other. Marx and Rostow use the term transition to address the path from beginning to end state, while Marx limits his use of the term transformation to micro-economic processes like the transformation of labor into wages and money into capital. Schumpeter distinguishes between periods of innovation and periods of non-innovation and does not consistently use the terms transition or transformation.

\subsection{Sociology}

In sociology the term transition is not normally cast. Contributions to the analysis of large scale and long term social change often use rise and fall or transformation in combination with a short description of a large scale social entity. Classic examples are Weber's account of the rise of capitalism in Europe (Weber 1904/1905) and of the emergence of rationalization and State and administrative bureaucracy (Weber 1925), Simmel's work on the societal impact of money, market and urbanization (Simmel 1904), Elias' analysis of the court society (Elias 1969) describing the fall of the agrarian feudal society and the rise of an urbanized industrial social system over a period of more than two centuries, and more recent, but not less classic, Wallerstein's works on modern-world system (Wallerstein 1974) and Castells' analysis of the rise 
of the network society (Castells 1996), which focuses on the development of the industrial society into a network based information society. Via Castells we also find our way back to complexity theory. According to Castells:

"at the end of the twentieth century, we lived through one of these rare intervals in history [punctuated events that occur with great rapidity with reference to Gould (1980); authors]. An interval characterized by the transformation of our 'material culture' by the works of a new technological paradigm organized around information technology".

Probably the most exact and consistent typology of social change in sociology can be found in the work of Gudmund Hernes, who distinguishes simple reproduction, extended reproduction, transition and transformation as basic patterns of social change (Hernes 1976). To draw up his typology Hernes characterizes social system by their functional form, parameter structure and output structure. With functional form Hernes indicates the structure and shape of the generating social process, while the parameter structure designates the variables that fit the functional form to a specific contingent situation. The output structure than describes the resulting social reality. For example in demography the functional form is described by the relation between survival probabilities and age-specific birth rates, the parameter structure than gives the values of these survival probabilities and age-specific birth rates for a specific time and social system while the output structure is the resulting population pyramid (Hernes 1976).

According to Hernes under simple reproduction the social system reproduces its functional form, parameter structure and output structure; e.g. both the total population and the population pyramid of a social system are stable. Under extended reproduction the output of the social systems changes, while its functional form and parameters structure are maintained; e.g. the population pyramid and total populations do change, not because the functional form or parameter structure changes, but because the population is not at equilibrium. Transitions entail a change in the parameter structure and the related change in output structure; e.g. the demographic transition in Europe caused by changes in survival rates and age-specific birth rates. In social transformation all three structures change; e.g. the introduction of the concept of migration into a social system.

In addition to his structured definition of social change, Hernes supplies us with three requirements of models describing structural change. According to Hernes these models should be able to explain constancy as well as change, must combine microand macro level analysis, and must encompass endogenous sources of change (Hernes 1976).

According to Squazzoni, to understand transitions, social scientists have used three types of theoretical constructs: dichotomies, synchronic theories, and historical diachronic models (Squazzoni 2008). Dichotomies are extrapolations of polar types of social systems (Cox 1974). The purpose is, for instance, to explain the difference between modern and pre-modern societies by abstracting and comparing the differences in their respective constituencies. In this case, a societal transition may be viewed as the process through which a given society adheres to one of the polar types. Synchronic theories aim at illustrating the general functioning and composition of societies as social systems. The most influential example is the famous Parsons model 
of traditional/modern societies, transitioning through differentiation and integration of social sub-systems (Parsons 1966, 1971). Historical diachronic models focus on the analysis of processes of change over time, sometimes with the identification of ideal-typical stages and dynamics. These models allow us to focus on processes of societal changes and specify the hidden mechanisms behind their emergence. Much of the descriptive work in transitions studies uses this construct (Driel and Schot 2005; Geels 2006; Schot et al. 1994; Verbong and Geels 2006).

Besides introducing the three theoretical constructs described above, Squazzoni connects sociology, more specific social simulation, and transition studies via complexity theory by pointing at the importance of micro-macro generative mechanisms and the emergence of transitions patterns from agent interaction (Squazzoni 2008).

In conclusion, sociology mostly describes structural change of entire societies and does not have the functional orientation of the societal transitions studied in transition studies, except for the functioning of the entire society itself. While transition studies analyses transitions as resulting from interconnected change in economical, technological, institutional, ecological, cultural, behavior and believe systems, sociology analyses this process of interconnected change itself.

\section{Computational and mathematical approaches}

\subsection{Analytic framework}

The overview of the use of transitions and related concepts in the foregoing section leads us to some interesting observations that are also relevant for mathematical or computational approaches to societal transitions. These observations are related to the implementation of the micro-macro link, a focus on both stability and change, the balance between quantitative and qualitative properties of social change, the use of dichotomies, synchronic models or diachronic reasoning, the characterization of social change in terms of reproduction, extended reproduction, transition and transformation, the conceptualization of resistance to change and the intentional and normative interpretation of social change. In the following these concept are further developed in relation to transition studies.

Micro-to-macro. Besides in the literature on social simulation, explication of the micro-to-macro link and concepts from complexity are virtually absent in the social science literature on structural change. Evolutionary economy is a notable exception. The theoretical framework used by evolutionary economics is firmly rooted in evolutionary theory from ecology and applies numerous related concepts from complexity theory like emergence and co-evolution. Transition studies conceptualize a micro, meso and macro level. The interaction between these levels is however only metaphorically substantiated as modulation and as such an operationalization of the micro-to-macro link is lacking.

Stability and change. In the policy sciences the alternation of stability and change is a central issue. Policy scientists not only intend to explain stability but also aim to 
explain change and in particular the fact that policy development is episodic. Sociology is mostly concerned with the "rise and fall" while political sciences emphasize both stability and change by pointing at the social conflict behind transitions and the stabilizing power op political elites. Organizational sciences and change management through their normative and intentional paradigm focus on change and conceptualize stability as some equilibrium or tranquil period without explaining how this equilibrium or tranquility is maintained. In transition studies these periods are described as dynamic equilibria; the pre-development preluding the next transition. Stability itself is not an issue in transition studies.

Quantitative and qualitative. Different branches of social science also differ in the appreciation of quantitative and qualitative change. Quantitative change is normally described using slow, incremental, equilibrium, stable, episodic and abrupt, while qualitative change is characterized as structural, system, institutional, dramatic or innovative. The policy sciences, but also some sociologists, emphasize the relative speed of change, while economics, management sciences, transition studies, political science and the majority of sociological theorizing emphasize the qualitative dimension of change. Transition studies emphasize both the speed of change and its structural character, while a relation between both is implicitly hypothesized but under conceptualized.

Type of reasoning. The use of dichotomies, synchronic or diachronic theoretical constructs can be attributed to some of the social science approaches to transitions described. For example in the organizational sciences, the archetypes of organizational transitions developed by Miller and Friesen (Miller and Friesen 1984) clearly use dichotomic theorizing in their description of the archetypes as a transition between two polar types of organizational systems. This approach, however, lacks a historic necessity because of its contingent methodology. Similarly the reframing model proposed by Lewin and applied by Fiol et al. suggests a dichotomy between a set of social values at the start of the transition and a new set of social values after the transition, in between the change process moves through the phases of frame braking, frame moving and frame re-aligning (Fiol et al. 1999; Lewin 1951). Of cause dichotomic reasoning is also at the hart of economic history with Marx and Rostow as leading examples.

Policy scientists mostly apply diachronic reasoning. However, their inclination to emphasize perturbations or disaster from outside the policy system carries a definite synchronic flavor. It seems that diachronic reasoning in the policy sciences is rather the result of a narrow demarcation of the research object, a policy system and not a social system, than the result of theoretical scrutiny.

Transitions studies seem to combine al three theoretical constructs. First, dichotomic reasoning is used in conceptualizing a start and end state before and after a transition, combined with an increase in sustainability. Second within the levels of the multi-level picture (Fig. 1) diachronic theorizing is applied, while, third, in the description of the interaction or modulation of niche, regime and landscape levels synchronic reasoning prevails. 
Patterns of change. A clear and consistent definition of structural change seems to be lacking in the separate disciplines, leaving the meaning of what is consistently labeled structural change up till now in this paper at a meta level even more incomprehensible. The typology presented by Hernes is a possible way out of this standstill. By distinguishing reproduction, extended reproduction, transition and transformation based on a description of social systems in term of their parameter structure, functional structure and output structure, Hernes arrives at a consistent and complete typology of patterns of social change (Hernes 1976). However, the exact approach of Hernes can not easily be applied to transition studies because the multi-level and multi-phase models of transition studies lack formal precision. Using a more metaphoric approach we can however conclude that a transition in the terminology of Hernes is equal to a societal transition in transition studies. In a societal transition, the functional structure, e.g. the function performed by the system for society at large, remains similar. The way this function is fulfilled, the output structure, changes dramatically and so does its parameter structure.

Resistance to change. The conceptualization of a resistance to change also leads to a methodological differentiation in empirical analyzes. While strands of social science that conceptualize resistance to change share a methodological focus on the historic condition of the system, theories that do not conceptualize resistance share a focus on the future stability of the system or a prescribed linear development path. Transition studies combines these viewpoints and explicitly take both the historic and future conditions of the societal system into account.

Intentionality and normativety. It seems that both the use of the term transition and the conceptualization of "resistance to change" or a need for "unfreezing" are connected to planned or intentional change. This gives to these transition approaches a normative flavor. As a consequence resistance to change or the need for unfreezing are not viewed as intrinsic characteristics of social systems, but are only mobilized when a direction for change is projected onto the system. This hypothesis is in line with a conceptualization of social systems as complex adaptive systems, systems that are capable of adaptation while resisting prediction and steering.

Sociologists, mainstream economists and the majority of policy scientists see transition like change as resulting form independent, unplanned, unintended or linear processes and use concepts like transformation, rise and fall and abrupt and episodic to represent these changes. Transition studies and change management explicitly use the concept of transition in relation with intentional change or management of change, while Marxism and development economics combine a perceived historic necessity with a normative flavor. It is also in these branches of the social sciences that an explicit focus on resistance to change is important. According to Marx the class structures has to be broken down, while development economics is concerned with barriers to and conditions for the transition to a modern market economy. Change management emphasizes the need for "unfreezing" of both organizations and their individual members. In historic economy, Schumpeter identifies the need for the entrepreneurs to harness themselves against conservative forces (Schumpeter 1912). Transition studies endow regimes with the ability to resist change (Rotmans 2005). 
This special issue brings the reader a bundle of articles with the common denominator of a computational or mathematical approach to societal transitions. Each article is a scientific contribution in itself and a contribution to the emerging research field. In the next section the articles in this issue are introduced and than described and compared using the theoretical constructs derived from the social science literature on structural change described above.

\subsection{Contributions in this issue}

In the section before a framework for the description and comparison of theories and models of structural social change was developed. This framework consists of seven concepts; implementation of the micro-macro link, focus on stability and change, balance between quantitative and qualitative properties of social change, employing dichotomic, synchronic or diachronic reasoning (Squazzoni 2008), characterization of social change in terms of reproduction, extended reproduction, transition and transformation (Hernes 1976), conceptualization of resistance to change and intentionality and normativety of social change.

In the following these concepts are used to describe and compare the model contribution in this special issue. With this exercise we aim to arrive at a common language enabling a discussion of transition models, arriving at a better understanding of what actually entails a transition model. An overview of the discussion in this section is presented in Table 1.

Schilperoord et al. "Modeling Societal Transitions Using Agent Transformation" provides a nice example of how the new subject matter of societal transitions can enrich existing methods, e.g., agent-based modeling. Basing themselves on the transition studies concepts of regimes and (empowered) niches, they directly interpret these as agents with different strategies. The agents can transform if they gain enough support or power and in this manner transition paths are produced. Since the modeler has control over the landscape signals and the strategies employed by the agents one gains insight in how transition paths are influenced. By introducing agent-transformation and having populations of simple agents and aggregate agents, this article also provides an innovation to the field of agent-based modeling.

Their model evolves endogenously as well as in reaction to an environment and the micro-macro link goes via the support simple agents give to the aggregate agents, which in turns strengthens and makes them more attractive. The idea of the societal system having a tendency to maintain equilibrium and thus resisting change is reflected in the different strategies for the aggregate agents, for instance regime agents employing a conservative strategy as opposed to niches taking more risks.

When applying the typology of Hernes to this model, we conclude that the model simulates up to transformative change, in which parameter structure, output structure and functional structure are adapted. Transformation is however limited by the predetermined output dimensions. The model applies diachronic reasoning in the modeling of the development of actors and aggregated actors, while synchronic reasoning is applied in the modeling of the interaction between these entities.

De Haan in "The Dynamics of Functioning: Investigating Societal Transitions with Partial Differential Equations" takes on an almost literal translation of the termi- 


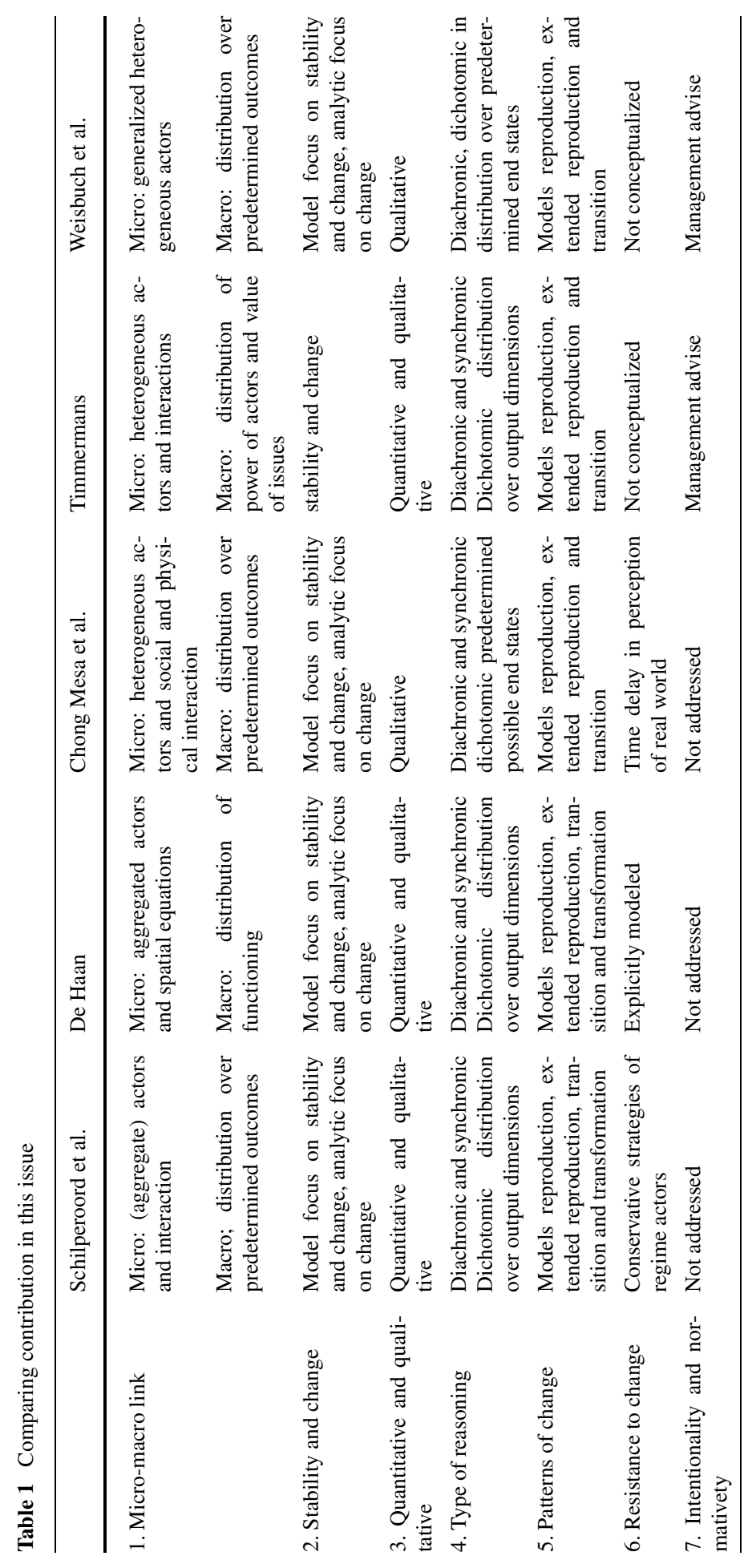


nology of transition studies into partial differential equations. Transitions are conceptualized as a significant change in the functioning of a societal system. For this, the regime and niches become fields that obey coupled partial differential equations. Within this framework the scaling up of niches is studied, both on direct competition with the incumbent regime and in the situation of a changed environment. Mathematically this is essentially an application of methods from the field of pattern formation and front propagation, whereas in the context of transition studies connections are made with notions as the scaling up of niches, empowerment and strategic niche management (Kemp et al. 1998).

The qualitative change, here in terms of societal functioning, one associate with transitions is parameterized in this modeling framework. This allowed the author to identify a measure for transitional change in his systems. The model works with an environment, which is conceptualized as societal support similar to the model by Schilperoord et al., (this issue) that influences the development of the system. Apart from this the model evolves endogenously under mechanisms of competition. The idea that the current societal status quo resists change is a parameter in the equations and its influence is studied. Although the model does not incorporate actors, or a clear system level, the fact that the equations are spatial introduces a micro-macro link. The micro level corresponds to specific forms of how a societal need is met which is connected to a macro level of the power of the regimes and niches at play.

In the model a transition is defined as a niche taking over the larger share of the societal function of the regime. In this process the qualitative functioning can change or not change, depending on the details of the model dynamics determined by initial conditions, parameters initialization and the specification of societal support. In a transition as defined in this model both the parameter structure and the functional structure of the regime are replaced by the parameter and functional structure of a niche. The related change in output structure is however not mandatory because the former niche can mimic the functioning of the former regime. The model is thus in principle able to simulate up to transformative change.

The model applies both diachronic reasoning and synchronic reasoning. Diachronic reasoning is found in the diffusion and growth dynamics of the separate niche and regime fields while synchronic reasoning is found in the interaction between niche and regime.

The contribution "Studying Transition Dynamics via Focusing on Underlying Feedback Interactions" by Chiong Meza and Yücel explores the potential of system dynamics for constructing "dynamic hypotheses" on the unfolding of (historical) societal transitions. System dynamics is normally applied to construct causal models of dynamic systems while human agency is reduced to the causal influence of generalized actors. In their paper, Chiong Meza and Yücel extend system dynamics to the social by integrating agency in the form of actors taking decisions. They apply their framework to develop a quantitative dynamic hypothesis for the transition in Dutch waste treatment from land filling to incineration and show that their modeling framework strengthens empirical research in the tradition of socio- technical systems research (Geels 2005, 2006; Verbong and Geels 2006), while taking transition theory's hypotheses on the importance of the interaction between social and non-social objects in societal systems seriously. The modeling framework presented paves the 
way for more abstract quantitative research on metaphorically strong concepts like path dependency and co-evolution used in transition theory and transition management. In short, Chiong Meza and Yücel develop "agent based system dynamics" as a promising tool for studying societal transitions.

The explicit modeling of the interaction between a 'real world system' and the social system sets this model apart from other approaches in this issue and facilitates the definition of transitional change both in a qualitative and quantitative sense. The authors use a definition of societal transitions that fits their theoretical framework, as it is mainly substantive and empirical. The model is also distinct in its explicit conceptualization of resistance to change as a heterogeneous time delay in the perception of new information by the actors, causing their rational behavior to be bounded. Unlike De Haan and Schilperoord et al., in this model the environment in which actors operate is endogenous and reacts to the decisions of the actors in the system. In its implementation of the micro-macro link, the model of Chiong Meza and Yücel is similar to agent based models. However, in the current implementation the conceptualization of the micro level is limited to aggregate actors adhering to a single value function. The models primarily applies diachronic theorizing, however, the a priori definition of alternative actions and their attributes gives it a dichotomic flavor, as both the start and end states of the model are limited to a pre-specified set of alternatives. In terms of Hernes' typology of patterns of social change, the system dynamics model of Chiong Meza and Yücel deals with transitional change. This is true both for the social and the physical sub-systems. For the model of the physical system this is straightforward. For the model of the social system, this conclusion is sustained by the fixed shape of the value functions of the actors and the predetermined structure of the interaction between the actors and between actors and the physical sub-system. In order to model transformative change, endogenous addition of new components to the component value functions of the actors, the introduction of novel alternative decisions or new insights into the functioning of the physical system are required.

Timmermans' article "Punctuated Equilibrium in a Non-linear System of Action" brings Coleman's Linear System of Action (LSA) into the realm of non-linear dynamics by adding a variation and selection algorithm that is consistent with the strong rational actor approach of the LSA. This Non-linear System of Action (NLSA) is proven to operate in the realm of organized complexity as shown by the power law distribution of the rate of social development. Model behavior supports hypotheses on the punctuated behavior of social systems found in the policy sciences and transition literature. In the realm of the Non-linear System of Action, transitions are defined as robust changes in the power distribution and/or the value structure of the social system and also address their relation. In this respect Timmermans further develops and conceptualizes the relation between qualitative and quantitative change hypothesized by transition studies.

The model incorporates only endogenous mechanisms and does not rely on developments outside the model to drive or direct social dynamics. The model is self contained and intentional steering is not conceptualized, nor is resistance to change. In addition to the micro-macro link established trough the exchange of control in the LSA, the variation and selection mechanism implemented in the NLSA operationalizes a second micro-macro link. This links connects the influence and interests of 
individual actors to potential for exchange of control, a systemic macro level variable. As a result the model not only shows dynamic development of the distributions of control over actors, but also of the interest of actors in issues. The punctuated equilibrium dynamics of the model probably results from the combination of these two mechanisms.

In terms of Hernes' typology, social change in the model can be characterized as transitional. The model dynamics cause development of both the parameter structure, the initial distributions of interests and control, and the output structure, the dynamic development of the distributions of interest and control. However, its functional form, described by a Cobb-Douglass type utility function with control and interest as parameters, remains unchanged. Modeling transformative change would require the endogenous addition of actors or issues to the NLSA a feature that is not included in the current version of the model.

In the contribution of Weisbuch, Buskens and Vuong, "Heterogeneity and Increasing Returns May Drive Socio-Economic Transitions", the authors practice the art of keeping it simple. They investigate how an initially economically disadvantaged green technology might still obtain the larger market share, which is in essence a study of a possible technological transition. They present a simple mathematical model based on a mechanism of increasing returns and find that under very basic assumptions such a model already exhibits complex behavior, such as regime shifts and hysteresis. They also connect their findings to possible policy interventions that could support such a transition. The article is a novel application of equations and principles well known in physics and economics, which in this form sheds new light on some mechanisms underlying transitions.

In this model the micro-to-macro transition is conceptualized as consumer choices resulting in a distribution of product shares over a set of predetermined types of products. These products draw up a multi-dimensional output space and the outcome is an attractor in this space and depends critically on initial conditions and parameter values. The models allows for a change in parameter values, for example increasing return to scale for product prices and a change in output structure. However, the functional structure, e.g. the equation themselves, remain the same. In the typology of Hernes this indicates that the model by Weisbuch, Buskens and Vuong simulates up to transitional change. Modeling transformative change would require the introduction of new products or additional preferences of the actors.

In relation to intentionality and normativety of social change, the model allows the authors to derive some valuable advises for policy makers or producers willing to force or inhibit a transition. In fact the authors show that analyzing policy measures as affecting a non-linear system could provide decision-makers with valuable insight and levers for influencing societal development that can not be obtained from traditional linear analysis.

\section{Discussion and outlook}

The central reason why modeling and simulation are important in the study of societal transitions is that these last are complex phenomena. Many mechanisms can influence 
the dynamics of transitions and the data describing transitions are multi-dimensional. In such situations, modeling gives the scientist grip and allows her or him to see if the hypotheses hold and if the observed patterns in the data can be explained by them. This is not to say that narrative approaches to explanation of transition phenomena are inferior. In some senses a narrative can capture complexity better than a model can. Rather, it provides an added value to such approaches if they can be framed in models. If modeling, theorizing and empirical research could be integrated more, the field would gain a lot of momentum.

This article has explored several questions: What are societal transitions? What do existing scientific fields and disciplines to say about them? Why do computational and mathematical approaches appear necessary? After that, an overview of the articles in this special issue that are about such methods was given. The task the article put for itself can be summarized as mapping the charts of the role of computational and mathematical approaches in a yet unknown but emerging scientific discipline: transition science.

In the section "Societal Transitions" the image appeared of a young field that is beginning to take shape. A field struggling with its theoretical foundations and its theoretical grounding in diverse areas as innovation studies, sociology and complexity theories. This in itself was argued to be a reason to employ computational and mathematical approaches, since the practical form of these approaches would force researchers to be conceptually more rigorous.

Nevertheless transition studies are at least converging to a view of transitions as structural and relatively abrupt change in societal systems, changing the very structures, cultures and practices that are at the basis of its functioning. This combined with the idea that there is a regime, embodying the status quo before the transition, and societal innovation that can emerge in niches is the basis of a language where a societal transition is a regime change. How such a regime change takes place and under what conditions is then the fundamental research question in transition science.

In the section "Structural change in social systems" the emergent theoretical bases of transition science was compared with how other scientific fields treat structural change in social systems. As expected the variety in approaches was enormous and common denominators only to be found on the most abstract level. It was interesting to learn that resistance to the transitional change was apparently of more concern to theories with a normative edge or a preoccupation with steering, something that has consequences of course for modeling approaches and their appreciation. What also varied wildly was on what aspect of a transition the theory focused; the inevitability of a new qualitative end state or the dynamic development and rate of change of some indicator of the transition, or both. The richness of theoretical constructs to model resistance to change, direct, time delay and conservative strategies came as a surprise and it is worthwhile to follow up this issue in more detail.

In the section "Computational and Mathematical Approaches" the question was asked what a transition model entails. To come to grips with this question an analytic framework based on theoretical constructs from the social science literature on structural change was developed and used in this article. These concepts are the implementation of the micro-macro link, a focus on both stability and change, the balance between quantitative and qualitative properties of social change, the use of 
dichotomic, synchronic or diachronic reasoning, the characterization of social change in terms of reproduction, extended reproduction, transition and transformation, the conceptualization of resistance to change and the intentional and normative interpretation of social change.

It is interesting to see that all models in this special issue have an approach where micro mechanisms lead to macro changes. Most apparent in articles such as Schilperoord et al. and Yücel and Chiong Meza, but even in the articles by Weisbuch et al. and de Haan, the transitions are studied on a level that is qualitatively higher then the mechanisms that drive their models. The agent based or agent based like models of Schilperoord, Chong Mesa and Yücel and Timmermans implement the micro-tomacro link as described by Coleman (Coleman 1990), however, Schilperoord et al. introduce an additional level of aggregate actors and endows actors with contingent strategies. Weisbuch, Buskens and Vuong employ actors as consumers on the micro level endowed with heterogeneous preferences. Their macro level outcome is a dynamic equilibrium distribution of consumer choices, that is, an attractor of the nonlinear system.

Off course all models in this special issue analyze and address change. The way stability is treated in the models is, however, less clear. It seems that all models are able to simulate periods of stability, but that the focus on change kept the authors from analyzing the conditions for stability to appear. An analysis of the conditions for stability could potentially offer a wealth of insight.

All models do deal with qualitative and quantitative societal change in more or less detail. In De Haan's partial differential equations approach, quantitative change is not conceptualized as a rate of change but as the share of a niche or regime in the total functioning of the societal system. The model is, however, able to simulate qualitative change, but remains at an abstract level and processes of qualitative change are not included in the model equations. Schilperoord et al. and Chong Mesa and Yücel focus on qualitative change described in terms of predetermined dimensions that are part of the model formulation. The model by Timmermans does model quantitative change in terms of a rate of social change and qualitative societal change in terms of power of actors and values of issues. In addition the description of qualitative change remains general, which is both a strength and a weakness of this model.

It seems that most of the models apply both synchronic and diachronic reasoning. Dichotomic approaches in the sense of a necessity of social development to reach one of the polar types of end states envisioned is not applied as a primary explanatory factor. However, all models more or less limit the dimensionality of their outcome space to a set of predetermined dimensions. Chong Mesa and Yücel and Weisbuch et al. are most restrictive in this sense, while de Haan, Schilperoord et al. and Timmermans offer a higher dimensionality of their output space.

All models in this special issue are able to model transitional change as defined by Hernes (Hernes 1976). This means that model dynamics both result in a change of the parameter and output structure of the social system simulated. De Haan comes closest to modeling transformative change because the field describing the dominant functioning at the onset of the simulation can be replaced by the entirely different field of an incumbent niche. The functional structures of these fields however are again predetermined by the modeler. 
The conceptualization of resistance to change in the models of this special issue is surprisingly diverse. In Chiong Meza and Yücel resistance to change takes the shape of a time delay in the perception of changes in the physical system by the actor in the social system. De Haan incorporates resistance to change directly in the partial differential equations describing the societal system. A third conceptualization of resistance to change can be found in Schilperoord et al. In this model resistance to change is a relative notion reflected in different strategies for the aggregate agents, for instance the regime agent employing a more conservative strategy as opposed to niches taking more risks.

None of the models is concerned with normative aspects of societal transitions. Although all models investigate or are suitable to investigate the conditions under which transitions prevail the direction of this change is not analyzed. Timmermans and Weisbuch et al., however, hint at the possibility of applying their models to study intentional and normative transitional change as proposed by transition management (Loorbach 2007). Weisbuch et al. even go one step further and give some examples of possible practical advises, when they point at the importance of timing in applying subsidies in order to intentionally bring about normative social change. In fact their advice is more general and suggests decision-makers to analyze their systems as nonlinear and use the wealth of opportunities these systems offer to increase the impact of their decisions.

Conversely one could observe that each article pushed the boundaries of their approaches a bit in applying them to transitions. Agent-based modeling was innovated with agent transformations and landscape signals by Schilperoord et al. System dynamics was given agency by Chiong Meza and Yücel. Timmermans complexified Coleman's linear system of action into a nonlinear one. Even the articles that stayed relatively close to their methodological roots did this by freely cross-fertilizing their methods with others; de Haan treated transitions as a nonlinear dynamics and pattern formation problem and Weisbuch et al. explored the paths of a technological transition combining methods from physics and economics.

The matter remains of the emerging research field and the role of this special issue in it. In any case the very existence of this special issue proves that the topic of societal transition invites researchers from various disciplines and trained in various methods to put their creative curiosity to work. Apparently something is happening. Something that sparked new ideas and collaborations that demanded to be elaborated in this special issue.

For a new research field to take shape however, it is necessary that at a certain point the research converges to more than just various methods applied to a common phenomenon under study. That is not to exclude beforehand the value of any approach, but similar to how a common conceptual language is arising slowly. In other words there has to emerge a more refined idea of what is transition modeling and what are computational and mathematical approaches to societal transitions. It is to this that this special issue not only hopes to have contributed, but also has provided a basis. 
Open Access This article is distributed under the terms of the Creative Commons Attribution Noncommercial License which permits any noncommercial use, distribution, and reproduction in any medium, provided the original author(s) and source are credited.

\section{References}

Aassve A, Billari FC, Spéder Z (2006) Societal transition, policy. Changes and family formation: evidence from Hungary. Eur J Popul 22(2):127-152

Aghion P, Howitt P (1992) A model of growth through creative destruction. Econometrica 60(2):323-351

Arthur WB (1994) Increasing returns and path dependence in the economy. University of Michigan Press, Ann Arbor

Baumgartner FR, Jones BD (1993) Agendas and instability in American politics. University of Chicago Press, Chicago

Berkhout F, Smith A, Stirling A (2004) Socio-technical regimes and transition contexts. In: Elzen B, Geels FW, Green K (eds) System innovation and the transition to sustainability. Edward Elgar, Cheltenham

Black CE (1966) The dynamics of modernization. Harper, New York

Braudel F (1979) Civilization and capitalism, 15th-18th century, III volumes. University of California Press, Berkeley, Reprint edition 1992

Byrne D (1998) Complexity theory and the social sciences. Routledge, London

Byrne DS (2001) Understanding the urban. Palgrave Macmillan, Houndmills, p 240

Castells M (1996) The information age: economy, society and culture, volume I of the rise of the network society. Blackwell, Oxford

Coleman JS (1990) Foundations of social theory. The Belknap Press of Harvard University Press, Cambridge, London

Cox OC (1974) The problem of societal transition. Am J Sociol 79(5):1120-1133

Davis K (1945) The world demographic transition. Ann Am Acad Polit Soc Sci 235:1-11

Deutsch K (1961) Social mobilization and political development. Am Polit Sci Rev 79(5):493-514

Driel HV, Schot J (2005) Radical innovation as a multilevel process: introducing floating grain elevators in the port of Rotterdam. Technol Cult 46(1):51-76

Eisenstadt SN (1973) Tradition, change and modernity. Prentice Hall, Englewood Cliffs

Eldredge N, Gould SJ (1972) Punctuated equilibrium: an alternative to phyletic gradualism. In: Schopf, Freeman TJF (eds) Models in paleobiology. Cooper \& Co, San Francisco, pp 82-115

Elias N (1969) The court society. Pantheon, New York

Fiol CM, Harris D, House R (1999) Charismatic leadership: strategies for effecting social change. Leadersh Q 10(3):449-482

Geels F (2005) Technological transitions and system innovations: a co-evolutionary and socio-technical analysis. Edward Elgar, Cheltenham, p 279

Geels F (2006) The hygienic transition from cesspools to sewer systems (1840-1930), the dynamics of regime transformation. Res Policy 35(7):1069-1082

Geels FW, Schot J (2007) Typology of sociotechnical transition pathways. Res Policy 36(3):399-417

Georgescu-Roegen N (1971) The entropy law and the economic process. Harvard University Press, Cambridge

Gilbert N (2008) Agent-based models. Sage, London

Gould SJ (1980) The Panda's Thumb: more reflections on natural history. W.W. Norton, New York

Gould SJ, Eldredge N (1977) Punctuated equilibria: the tempo and mode of evolution reconsidered. Paleobiology 3:115-151

Gunderson L, Holing CS (2002) Panarchy: understanding transformations in human and natural systems. Island Press, Washington, Covelo, London, p 507

Hernes G (1976) Structural change in social processes. Am J Soc 82(3):513-547

Jones BD, Baumgartner FR (2005) A model of choice for public policy. J Public Adm Res Theory 15(3):325-351

Kemp R, Schot J, Hoogma R (1998) Regime shifts to sustainability through processes of niche formation: the approach of strategic niche management. Technol Anal Strat Manag 10:175-196

Kingdon JW (1984) Agendas, alternatives and public policy. Longman, imprint of Addison Wesley Longman, New York 
Lewin K (1951) Field theory in social science. Harper and Row, New York

Linz JJ, Stepan A (1996) Problems of democratic transition and consolidation: Southern Europe, South America, and post-communist Europe. Johns Hopkins University Press, Baltimore

Loorbach D (2007) Transition management: new mode of governance for sustainable development. International Books, Utrecht

Medearis J (2001) Ideology, democracy and the limits of equilibrium: a Schumpeterian critique. Br J Polit Sci 31:355-388

Miller D, Friesen P (1984) A longitudinal study of the corporate life cycle. Manag Sci 30(10):1161-1183

Nicolis G, Prigogine I (1989) Exploring complexity. W.H. Freeman, New York, p 313

North DC (2005) Understanding the process of economic change. Princeton University Press, Princeton

Parsons T (1966) Societies: evolutionary and comparative perspectives. Prentice-Hall, Englewood Cliffs

Parsons T (1971) The system of modern societies. Prentice-Hall, Englewood Cliffs

Polanyi K (1944) The great transformation: the political and economic origin of our time. Beacon Press, Boston

Potts J (2000) The new evolutionary microeconomics: complexity, competence and adaptive behaviour. Edward Elgar, Cheltenham

Rammel C, van den Bergh JCJM (2003) Evolutionary policies for sustainable development: adaptive flexibility and risk minimising. J Evol Econ 47(3):121-133

Rip A, Kemp R (1998) Technological change. In: Rayner S, Malone EL (eds) Human choice and climate change, vol 2. Battelle Press, Columbus, pp 327-399

Rostow WW (1960) The stages of economic growth: a non-communist manifesto. Cambridge University Press, Cambridge

Rotmans J (1998) Methods for IA: The challenges and opportunities ahead. Environ Model Assess $3(3): 155-179$

Rotmans J (2005) Societal innovation: between dream and reality lies complexity. ERIM, Erasmus Research Institute of Management, Rotterdam, p 76

Rotmans J, Kemp R, van Asselt M, Geels F, Verbong G, Molendijk K (2000) Transitions and transition management, the case of an emission-free energy supply. ICIS/MERIT, Maastricht

Sabatier PA, Jenkins-Smith HC (1993) Policy change and learning - an advocacy coalition approach. Westview Press, Boulder

Schot J, Hoogma R, Elzen B (1994) Strategies for shifting technological systems. The case of the automobile system. Futures 24(10):1060-1066

Schumpeter JA (1912) The theory of economic development: an inquiry into profits, capital, interest, and the business cycle. Transaction Publishers, New Brunswick and London

Schumpeter JA (1942) Capitalism, socialism and democracy. Harper, New York

Simmel G (1904) The philosophy of money. Routledge, London

Squazzoni F (2008) A (computational) social science perspective on societal transitions. Comput Math Organ Theory (this issue)

van den Bergh JCJM, Gowdy JM (2003) The microfoundations of macroeconomics: an evolutionary perspective. Cambridge J Econ 27:65-84

van den Bergh JCJM, Kemp R (2006) Economics and transitions: lessons from economic sub-disciplines. UNU-MERIT working paper series 2006-038. http://www.merit.unu.edu/publications/wppdf/2006/ wp2006-038.pdf

van den Bergh JCJM, Stagl S (2006) Co-evolution of economic behaviour and institutions: towards a theory of institutional change. J Evol Econ 13(3)

van der Brugge R, Rotmans J (2006) Towards transition management of European water resources. Water Res Manag 21(1):249-267

Verbong G, Geels F (2006) The ongoing energy transition: lessons from a socio-technical, multi-level analysis of the Dutch electricity system (1960-2004). Energy Policy 35(2):1025-1037

Wallerstein I (1974) The modern world-system I. Capitalist agriculture and the origins of the European world-economy in the sixteenth century. Academic Press, New York

Weber M (1904/1905) The protestant ethic and the spirit of capitalism. Routledge, London

Weber M (1925) Economy and society. University of California Press, Berkeley

Wilson CA (2000) Policy regimes and policy change. J Public Policy 20(3):247-274

Witt U (2003) The evolving economy. Edward Elgar, Cheltenham

Wolfram S (2002) A new kind of science. Wolfram Media, Champaign, p 1197

Worren NAM, Ruddle K, Moorem K (1999) From organizational development to change management: the emergence of a new profession. J Appl Behav Sci 35(3):273-286 
Jos Timmermans obtained his PhD from the school of Policy Analysis and Management of Delft University of Technology. He is currently employed as researcher and consultant with the Dutch Research Institute for Transitions of Erasmus University Rotterdam. He works from the punctuated equilibrium paradigm and his research interests include stakeholder analysis, policy entrepreneurship and social network theory.

Hans de Haan obtained his master degree in theoretical physics from the University of Leiden. He is currently employed as PhD researcher with the Dutch Research Institute for Transitions of Erasmus University Rotterdam. His primary research interests are the development of theory for, and computational and mathematical approaches to, societal transitions.

Flaminio Squazzoni is Assistant Professor of Economic Sociology (University of Brescia). His main field of research is social simulation. He is Review Editor of JASSS. 